Dev World Bioeth. 2008 December ; 8(3): 235-241. doi:10.1111/j.1471-8847.2007.00220.x.

\title{
TO TELL OR NOT TO TELL:
}

\author{
HIV DISCLOSURE TO FAMILY MEMBERS IN CHINA
}

LI LI, CHUNQING LIN, ZUNYOU WU, LYNWOOD LORD, and SHENG WU

\begin{abstract}
Laws in China relating to HIV disclosure are inconsistent. After a patient has tested HIV-positive, service providers struggle to decide who should be informed first: patients, family members, or both. To understand service providers' attitudes and practices regarding the HIV notification process in China, 1101 service providers from a southwestern province of China were surveyed. Opinions were gathered from providers at five different levels of health care facilities (provincial, city, county, township and village). A mixed methods approach was used to analyze perceptions of informing family members of a patient's HIV status. Quantitative analysis was used to examine whether providers held a favorable attitude toward notifying family members first and qualitative analysis was used to explore the reasons and consequences of notifying family members first. Nearly half of service providers felt family members should be informed of a patient's HIV status first. Providers who were older, had contact with HIV patients, or had less medical education were more likely to agree with a family-first notification practice. Psychological pressure, concern about protecting family members, the need for family support, and consideration for local regulations were cited as the main reasons for this practice. There is an immediate need to re-examine HIV notification policies so that there are consistent guidelines and procedures for providers throughout China.
\end{abstract}

\section{Keywords}

HIV/AIDS; China; disclosure; family; family members

\section{INTRODUCTION}

Wang is a 40-year-old male. In August 2003, he felt sick and went to the local hospital for a medical examination. He had blood tests there. Three days later, Wang was told to bring his wife and come again for a confirmatory test. A week later the doctor informed his wife that Wang was HIV-positive and suggested she have an HIV test. Under tremendous mental stress, Wang's wife went home and eventually committed suicide. Wang filed a law suit against the hospital. He believed that the hospital should not have informed his wife of his HIV status and the hospital's negligence led to his wife's suicide. He asked the hospital to take responsibility for the death of his wife. Court verdict: the hospital is not at fault regarding the test result notification and should not bear legal responsibility for his wife's death. ${ }^{1}$

This Chinese legal ruling reflects a new HIV-related dilemma for health care providers: protecting confidentiality and privacy for patients living with HIV (PLH) versus informing family members at risk of being infected. Some argue that confidentiality is the most effective way to get patients into therapeutic alliances and bring about better outcomes,${ }^{2}$ but many

(C) 2007 Blackwell Publishing Ltd.

Address for Correspondence: Li Li, UCLA-NPI Center for Community Health, 10920 Wilshire Blvd., Suite \#350, Los Angeles, CA 90024, USA. E-mail: lililili@ucla.edu. 
disagree, arguing that the duty of confidentiality should not be absolute. ${ }^{3}$ Resolving the issue is difficult as there are many inconsistencies in Chinese law. A similar challenge exists in other countries, including the United States. 4

In China's Law of Certified Medical Doctor, Article 22 stipulates: 'A health care provider has the responsibility to protect the patient's privacy. ${ }^{5}$ In the same document, it is also stated that health care providers should honestly inform the patient or his/her family member(s) about the condition of the disease. Article 42 in the latest AIDS Treatment and Prevention Regulation states: 'HIV-positive status should only be informed to the patient him/herself; for minors or incapable persons, HIV status should be told to his/her guardian. ${ }^{6}$ Yet Article 38 states: 'HIVinfected people and AIDS patients have the responsibility to tell their sexual partners of the illness. ${ }^{\text {t }}$ Given ambiguous and contradictory laws, it has become especially important to understand service providers' attitudes and practices regarding the HIV notification process in China.

Using both qualitative and quantitative approaches, we investigated service providers' attitudes and practices of informing family members of a patient's HIV status and describe their reasons for such attitudes and practices. We also examine the process of HIV test result notification and the perceived consequences of HIV status disclosure to family members.

\section{METHODS}

A self-administered questionnaire and interview guide were designed by the research team, local health educators, administrators, and providers. Data were collected between January and August 2005 from three study sites in Yunnan province, which has the highest number of reported HIV infections in China (40\% of all reported HIV cases). ${ }^{8}$

Both quantitative and qualitative analyses were used. The quantitative method consisted of a survey to determine the distribution and pattern of service providers' favorable attitudes towards notifying family members of a patient's HIV status. The qualitative arm of the study explored various reasons why service providers notified family members of a patient's HIV status while identifying the process and perceived consequences of this practice.

\section{Quantitative method: a representative survey}

Service providers working at different levels of health care facilities (provincial, city, county, township and village) were sampled using a two-stage method. In the first stage, three of nine provincial hospitals, four of seven city/prefecture hospitals, 10 of 35 county hospitals, 18 of 90 township health clinics, and 54 of 573 village clinics were randomly selected. In the second stage, the staff list of each facility was obtained. Doctors and nurses were proportionally sampled according to the doctor-nurse ratio and lab technicians were over-sampled to compensate for inadequate presentation in the analysis. A total of 1101 service providers voluntarily participated in the anonymous survey, with a refusal rate of less than $8 \%$.

The self-administered anonymous survey contained questions about demographic information, level of care (provincial, city, county, township or village), education (associate medical degree or not), profession (doctor, nurse, or lab technician), contact with patients living with HIV/ AIDS at work (yes or no), and HIV-related training (yes or no). A favorable attitude toward HIV disclosure to family members was measured by agreement with the following statement: 'Family members, not the patient, should be the first to be informed of a patient's HIV status by the provider.'

Descriptive distribution of age, gender, education, level of care, profession, and HIV training were crosstabulated with favorable attitudes toward family member notification. In order to 
examine independent associations between the favorable attitude of disclosure to family first and the selected variables, we further conducted multiple logistic regression analyses to control for simultaneous effect of other factors (age, gender, education, level of care, profession, HIV patient contact, and training). Adjusted odds ratios (aOR), 95\% confidence intervals and $P$ values are reported.

The study sample of the quantitative study was primarily female (74.4\%) and Han ethnicity (72.2\%), the ethnic majority in China. Approximately $26 \%$ of the respondents were younger than 30 years old and $29 \%$ were 41 years or older. More than $40 \%$ of the sample worked in provincial hospitals or city/prefecture hospitals. More than half (51\%) of the participants were doctors, $40 \%$ were nurses, and nearly $9 \%$ were lab technicians. At the time of the survey, about $59 \%$ of the sample had an associate medical degree or higher. Among all participants, $45 \%$ reported having contact with HIV-positive patients at work, and 68\% reported having received HIV-related training.

\section{Qualitative method: in-depth interviews}

From each of the five levels of care, facilities conducting the greatest amount of HIV testing and having the most HIV-positive patients were selected. From each facility, several doctors and nurses who had worked with PLH and who were familiar with the HIV notification situation of their hospital were invited to participate in the interview.

In-depth interviews took about two hours. Before the interview, interviewees were informed of the purpose of the study, procedures, and potential benefits and risks of participation.

Interviews were conducted with open-ended questions about HIV-related attitudes, perceived risk of occupational exposure and infection, and the process of HIV test result notification in their hospital. For those who held a favorable attitude towards family member notification, interviewers queried their reasons and perceived consequences of such practice.

A total of 33 interviews were conducted. All interviews were transcribed and cross checked by two different staff members. A first draft of the code list, which consisted of common themes discovered in the transcripts, was developed based on the interview guidelines and actual content of a number of interview transcripts. In order to refine the code list, the first transcript was coded by the entire research team. ${ }^{9}$ After several versions of the code list were created, a total of 62 codes and 14 code 'families' (a group of codes with the same theme) were developed. Analyses were conducted by identifying the themes occurring most frequently and putting them in the context of other information relayed by the participants.

\section{RESULTS}

Among the 1101 service providers in the study, $49 \%$ agreed that family members should first be informed of a patient's HIV status by the provider. Relationships between the providers' endorsement attitude and their demographic and HIV-related background are presented (see Table 1). A service provider's age, contact with HIV patients, and education were significantly associated with a favorable attitude towards the notification of HIV status to family members first. Specifically, participants who were older, had contact with PLH, or had less medical education were more likely to hold a favorable attitude towards family member notification; those who were younger, had no known contact with PLH, or had an educational level of associate medical degree or higher were less likely to have this attitude. No significant associations were found between the endorsement attitude and gender, level of care, profession, or HIV training.

Results from multivariate analyses with a multiple logistic regression model are summarized in Table 2. The relationship between a favorable attitude towards notification of HIV to family 
members and age, contact with HIV patients at work, and education remained significant after simultaneously controlling independent variables. Significant predictors of a favorable attitude towards HIV notification of family members were: age older than $40(\mathrm{OR}=1.68 ; P<0.001)$; previous contact with HIV-positive patients at work $(\mathrm{OR}=1.58 ; P<0.001)$; and less than an associate medical degree $(\mathrm{OR}=1.32 ; P<0.05)$.

\section{Reasons for informing family members}

Qualitative data analyses revealed four main reasons that service providers supported disclosing HIV test results to family members: (1) worry about psychological pressure associated with the news, (2) consideration of social culture and local regulations, (3) need of family support, and (4) concern about protecting family members.

Many providers compared HIV to cancer and treated HIV-positive patients similar to cancer patients. They believed that hiding the truth from the patient was protective and was better for the patient's physical as well as psychological status:

I am not sure whether they could accept it or not. Usually we will just tell the family member. In our hospital . . for example, we won't tell a cancer patient that he or she has got cancer. We only tell the family member. It is the same for HIV patient. [Female, 49 years old, nurse, internal medicine department, city level hospital]

Once the patient knows that he's got a disease ... maybe he will just go crazy with the test result. Just like cancer patients, before they knew the result, they might still feel well and cooperate well with our doctors and nurses. But once they know, it is possible for them to have a mental collapse. This is not good for their treatment. [Male, 37 years old, doctor, surgery department, township level hospital]

Some providers perceived that societal discrimination would impose a heavy psychological burden onPLH, while others worried that once patients knew their HIV status, they might take revenge on society:

We worry that the result is not acceptable for the patient. We only tell the family member now and there is strong discrimination towards people living with HIV in society. In order not to give them too much pressure, we choose not to tell them. [Male, 48 years old, doctor, internal medicine department, county level hospital]

Our concern is that the patient won't get over it, and will do something to get revenge on society. For example, someone will be like 'I got HIV from somewhere, it is reasonable for me to spread the virus to others.' Have you heard the news about the needle stick on the street? We are worried about this kind of thing. [Male, 47 years old, doctor, emergency room, provincial level hospital]

Some hospitals require that service providers get a family member's consent to inform the patient of their HIV status. Though in conflict with the law, these regulations have been regarded by service providers as appropriate protection for themselves and for hospitals to avoid lawsuits:

I know the law regulates that the patient should be informed of the test result. But you know culture varies from place to place. Another thing is, in our hospital, there is a rule that the family members need to sign a consent form indicating that they know the illness status of the patient. [Male, 45 years old, doctor, internal medicine department, provincial level hospital]

This is also a protection of our providers. Otherwise they will say that they got the virus from your hospital. [Male, 37 years old, doctor, infectious disease department, city level hospital] 
Another rationale providers reported was related to China's family oriented culture. It is likely that if a patient copes with HIV alone, he or she will not be able to afford HIV treatment and testing. Family members are expected to help with treatment costs and provide psychological support:

If nobody but the patient himself knows the situation, I think he will be very sad and desperate. In most cases, the patient needs the family's help and cooperation. [Female, 33 years old, doctor, internal medicine department, county level hospital]

In the process of giving test results, service providers suggested that family members, especially a spouse, take an HIV test. Also, providers would educate family members on how to protect themselves, thus preventing them from becoming infected:

If the spouse didn't know the HIV thing, they wouldn't use protection when they have sex [Female, 33 years old, doctor, internal medicine department, county level hospital]

For the family members, such as the kids and spouse, we strongly suggest they have an HIV test too ... We teach the family members the HIV/AIDS knowledge and protection. [Male, 45 years old, doctor, internal medicine department, provincial level hospital]

\section{Consequences of informing family members}

Although interviewees reported legitimate reasons for disclosing HIV status to family members, some also observed negative consequences of this practice. For example, some family members chose to discharge the patient after receiving the test results, mainly because they were unable to afford high treatment fees. Our interviewees also said that after HIV status was disclosed to family members, discrimination occurred within the family:

Some family members were so nervous that they discarded the patient's belongings. We explained the transmission routes to the family members. We told them that one couldn't be infected through daily living and dining together. But some family members still told me that they burned all of the patient's stuff. [Male, 29 years old, doctor, surgery department, city level hospital]

\section{Process of HIV notification to family members}

The qualitative data analyses also illuminated how HIV test results are relayed in local hospitals. Some interviewees described that they first made a subjective judgment of the situation to decide whether to inform a patient or his/her family members first. Judgments were based on patient's gender, transmission route, and illness stage. When service providers made a decision to disclose, they often chose a core family member such as a spouse or parent:

Usually we would judge the patient's acceptance of the disease. If he or she has a history of drug use or risky sex, we estimate that he or she already is prepared for the disease, and then we will tell the patient. [Male, 38 years old, doctor, infectious disease department, city level hospital]

In most cases, if the patient is an elder or female, we tend to tell their family members first, $\mathrm{hmm}$. . . ask their spouse or other family members' opinion. [Male, 38 years old, doctor, infectious disease department, city level hospital]

The service provider responsible for notifying patients varied from hospital to hospital. (Test results were delivered by department directors, doctors, lab technicians, or local CDC staff members.) As many local hospitals only had the capability to perform HIV screening tests, service providers often suggested that family members send a blood sample to the provincial CDC to have a confirmatory test. In these cases, family members got involved in the testing process even before the patient's HIV status was confirmed: 
We discuss with the family members about treatment in the future. We tell family members what kind of disease the patient has, what the stages are, and where to get treatment. If the family members mentioned that they want to get treatment in a certain hospital, we will provide help. [Male, 37 years old, doctor, surgery department, county level hospital]

After being notified of a patient's HIV status, family members must make the decision whether to tell the patient. If they decide not to tell the patient, they need to provide a signature (signed) approval, and the doctor helps to keep the result secret from the patient:

If the family members were willing to tell the patient, we would tell the patient. If they were not, they signed an agreement saying that they, the family members, didn't want the patient to know, so we will keep it secret [Male, 45 years old, doctor, internal medicine department, provincial level hospital]

If the screening test was positive, we wouldn't tell the patient directly. We asked family members their opinions first. If the husband thinks it is necessary, then we will tell the woman, if not, then we will just keep it secret. [Male, 34 years old, doctor, otolaryngology department, county level clinic]

\section{DISCUSSION}

This study presents a systematic investigation of service providers' attitudes and practices of HIV status notification to family members in China. Combining quantitative and qualitative methods, we learned not only the general patterns of providers' attitudes toward HIV disclosure to families, but also providers' reasons for their actions and the process of family disclosure practice.

Under the influence of Western culture, service providers and decision makers in China havegradually begun to recognize patient's rights in decision making and the issue of confidentiality. ${ }^{10}$ However, China has a long history of traditional medical ethics rooted in Confucianism, and significant differences between Chinese and Western cultures exist in clinical ethical practice. ${ }^{11}$ According to cultural tradition in China, individuals are viewed as embedded in the family and society. Following this tradition, for serious diagnoses or fatal prognoses, service providers usually first inform a close member of the patient's family. It then becomes the family's decision whether to tell the patient the truth. Service providers in this study followed similar procedures during the HIV disclosure process, choosing to hide the truth or even lie to respect what the family considered to be the best interest of the patient.

Previous studies have demonstrated that family support generates multiple levels of positive impacts on PLH. ${ }^{12}$ Participants identified specific reasons to support their decision to inform family members, including the need for support with psychological stress, treatment costs, and daily care for the patient. Despite these positive consequences of getting family involved in HIV disclosure and treatment, our study also revealed problems. In some families, disclosure resulted in family discrimination, which clearly added to patients' psychological burden.

HIV disclosure to family members is not just an ethical dilemma; it has important clinical implications. As more medications become available, service providers play a greater role in helping patients and their family members focus on treatment and care, rather than the 'fatal' nature of the disease. HIV treatment adherence requires a patient's full participation, and appropriate disclosure to patients is an important step in effective treatment. As there are no enforced policies in place for HIV status notification in China, service providers are trusted to use their judgment to choose the best method for test result notification. 
Some providers would rather avoid directly confronting the patient with the test results, putting the responsibility of further disclosure and counseling on the shoulders of family members. Although families can provide a buffer for patients when they are notified of their status, it is questionable whether they are equipped with sufficient knowledge to make a good decision about disclosure to the patient, given that the decision may have a huge impact on the PLH's future treatment and care.

There is an immediate need to re-examine the policies regarding HIV status notification in order to ensure consistent guidelines and procedures for providers throughout China. These guidelines should be culturally sensitive, with the goal of achieving balance between protecting individual privacy and maximizing family and social support for patients living with HIV/ AIDS. The policies and guidelines should be enforced by law and be fully implemented as a standard for medical practice in China. The lessons we learned from this study, that the policies and laws should be culturally appropriate, also have implications for other countries.

\section{Acknowledgment}

This paper was completed with the support of National Institute of Mental Health grant R01MH070931 and Fogarty International. We would like to thank Lin Lu, Manhong Jia, Zhihua Yan, Fan Zhang, Yong Zhang, Haixia Cui and other team members for their support and contribution to this study.

\section{References}

1. Liu, X. Informing HIV Status by Service Providers. China Medical Tribune - Online. 2000 [Accessed 29 Mar 2007]. Available at: http://www.cmt.com.cn/article/060629/a0606291804.htm

2. Kipnis K. A Defense of Unqualified Medical Confidentiality? Am J Bioeth 2006;6:7-18. [PubMed: 16500838]

3. Balint J. Should Confidentiality in Medicine be Absolute? Am J Bioeth 2006;6:19-20. [PubMed: 16500839]Capron A. Addressing an Ethical Dilemma Dialogically Rather than (Merely) Logically. Am J Bioeth 2006;6:36-39. [PubMed: 16500849]Hodge J. A Legal and Ethical Fiction of "Pure" Confidentiality. Am J Bioeth 2006;6:21-22. [PubMed: 16500840]Gibson E. Medical Confidentiality and Protection of Third Party Interests. Am J Bioeth 2006;6:23-25. [PubMed: 16500841]

4. Klitzman RL, et al. Naming Names: Perceptions of Name-based HIV Reporting, Partner Notification, and Criminalization of Non-Disclosure among Persons Living with HIV. Sex Res Social Policy 2004;1 (3):38-57.

5. People's Republic of China's Law of Certificated Medical Doctor; National People's Congress of China; 1999; [Accessed 7 Jan 2007]. Online: National People's Congress of China: Article 22. Available at: http://www.gov.cn/banshi/2005-08/01/content_18970.htm

6. State Council of the People's Republic of China. AIDS Treatment and Prevention Regulation. 2006 [Accessed 12 Feb 2007]. Online: State Council of the People's Republic of China: Article 42. Available at: http://www.gov.cn/zwgk/2006-02/12/content_186318.html

7. Ibid: Article 38.

8. State Council AIDS Working Committee Office. UN Theme Group on HIV/AIDS in China. A Joint Assessment of HIV/AIDS Prevention, Treatment and Care in China. State Council AIDS Working Committee Office, National Center for AIDS/STD Prevention and Control, China CDC \& UNAIDS China Office; Beijing: 2004 [Accessed 1 Sept 2007]. Available at:

http://www.casy.org/engdocs/JAREng04.pdf

9. Sandelowski M. The Problem of Rigor in Qualitative Research. Adv Nurs Sci 1986;8:27-37.

10. Hennig W. Bioethics in China: Although National Guidelines are in Place, Their Implementation Remains Difficult. EMBO Rep 2006;7:850-854. [PubMed: 16953195]

11. Feldman MD, Zhang J, Cummings SR. Chinese and U.S. Internists Adhere to Different Ethical Standards. J Gen Intern Med 1999;14:469-473. [PubMed: 10491230]Fan R, Li B. Truth Telling in Medicine: the Confucian View. J Med Philos 2004;29:179-193. [PubMed: 15371186]Chan HM. Informed Consent Hong Kong Style: An Instance of Moderate Familism. J Med Philos 2004;29:195- 
206. [PubMed: 15371187]Lapine A, et al. When Cultures Clash: Physician, Patient, and Family Wishes in Truth Disclosure for Dying Patients. J Palliat Med 2001;4:475-480. [PubMed: 11798479] 12. Li L, et al. Understanding Family Support for People Living with HIV/AIDS In Yunnan, China. AIDS Behav 2006;10:509-517. [PubMed: 16741672] 
Table 1

Distribution of the Endorsement of Disclosing HIV Status to Family Members by Demographic and Medical Experience

\begin{tabular}{|c|c|c|c|}
\hline & Total & $\begin{array}{l}\text { Endorsement of disclosing to } \\
\text { family members, } \%(\mathrm{~N})\end{array}$ & $\mathbf{P}$ \\
\hline Age & & & $<0.0001$ \\
\hline$<30$ years old & 321 & $42.37(136)$ & \\
\hline 31 to 40 years old & 453 & $47.02(213)$ & \\
\hline$>40$ years old & 313 & $58.47(183)$ & \\
\hline Gender & & & 0.8668 \\
\hline Male & 282 & $49.29(139)$ & \\
\hline Female & 815 & $48.71(397)$ & \\
\hline Level of care & & & 0.1796 \\
\hline Provincial & 250 & $42.80(107)$ & \\
\hline City & 198 & $53.03(105)$ & \\
\hline County & 400 & $52.25(209)$ & \\
\hline Township & 148 & $37.16(55)$ & \\
\hline Village & 102 & $59.80(61)$ & \\
\hline Profession & & & 0.1166 \\
\hline Doctor & 554 & $46.57(258)$ & \\
\hline Nurse & 439 & $50.80(223)$ & \\
\hline Lab technician & 94 & $53.19(50)$ & \\
\hline Contact HIV patient at work & & & 0.0039 \\
\hline No & 621 & $45.09(280)$ & \\
\hline Yes & 477 & $53.88(257)$ & \\
\hline Education & & & 0.0045 \\
\hline Less than associate degree & 444 & $54.05(240)$ & \\
\hline Associate degree or higher & 634 & $45.27(287)$ & \\
\hline HIV training & & & 0.0889 \\
\hline No & 360 & $45.28(163)$ & \\
\hline Yes & 737 & $50.75(374)$ & \\
\hline
\end{tabular}


LI et al.

Page 10

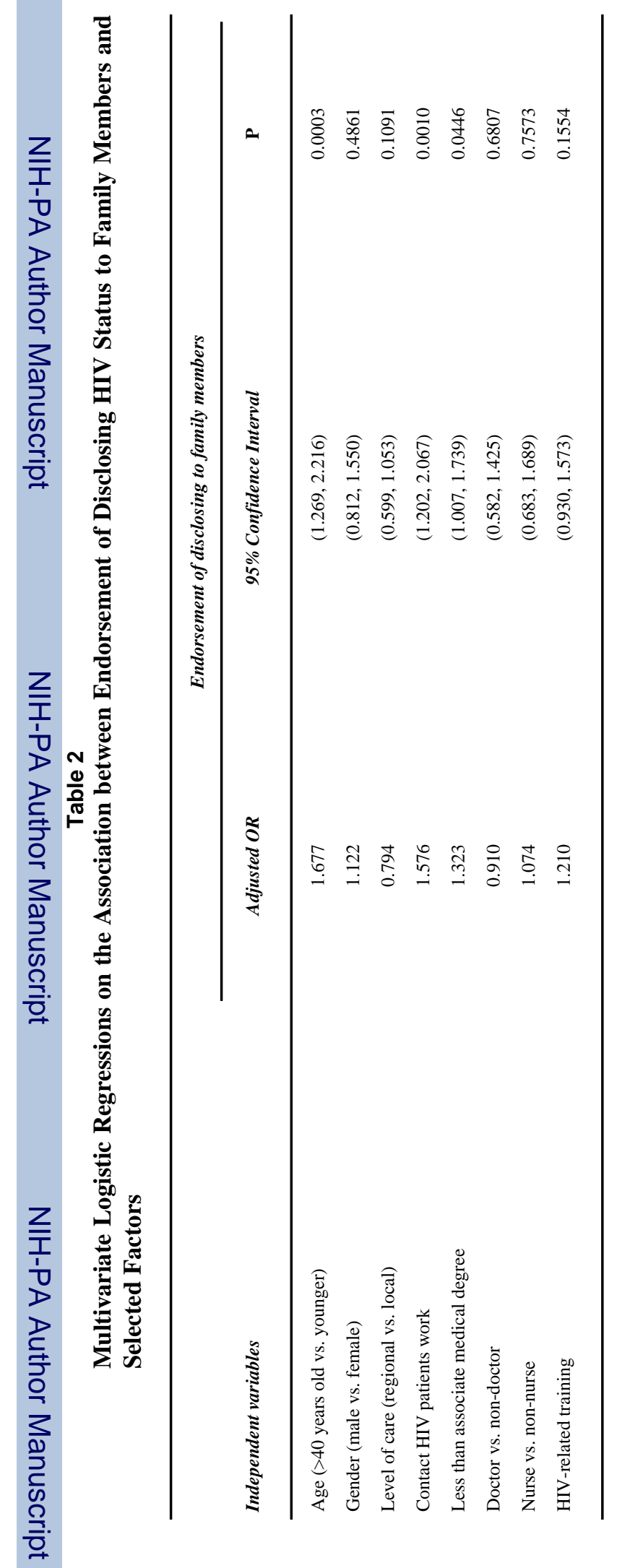

Dev World Bioeth. Author manuscript; available in PMC 2009 July 16. 